\title{
Automatic WBAN Area Recognition Using P2P Signal Strength in Office Environment
}

\author{
Joonyoung Jung *, Dongoh Kang*, Changseok Bae* \\ * Human Computing Research Section \\ Electronics and Telecommunications Research Institute, Deajeon, Korea \\ jyjung21@etri.re.kr, dongoh@etri.re.kr, csbaedetri.re.kr
}

\begin{abstract}
The distance estimation between mobile devices is a fundamental issue for a lot of applications of indoor wireless body area network (WBAN). The RSSI have been used to estimate the distance based on the received signal strength from another mobile device. Theoretically, the signal strength is inversely proportional to squared distance, and there is a known radio propagation model that is used to convert the signal strength into distance. However, in real environments, it is hard to measure distance using RSSI because of noises, obstacles, and the type of antenna. Distance estimation using RSSI in real-world applications is still questionable because of inaccuracy. However, RSSI could become the most used technology of distance estimation from the cost/precision viewpoint because of low cost. Mobile devices need to recognize each other in office environment automatically. However, distance estimation using the RSSI of Bluetooth is difficult because of large deviation of RSSI value. This paper provides the experimental results of RSSI measurement between mobile devices in office environment. And it applies the Low Pass Filter (LPF) to reduce the deviation of RSSI value. This paper shows that the distance estimation to recognize WBAN area is possible clearly when Bluetooth RSSI LPF data are used.
\end{abstract}

Keywords — Bluetooth, RSSI, Distance estimation, WBAN, Office environment.

\section{INTRODUCTION}

There are many applications for Wireless Sensor networks (WSNs) in military and civil applications [1]. The distance estimation between mobile devices is a fundamental issue for lots of applications of indoor WSNs, such as a Bluetooth and Zigbee. Distance estimation identifies the distance between two devices in wireless network. Such estimates are also an important component of localization systems, because they are used by the position computation and localization algorithm components. Different methods, such as RSSI, time of arrival (ToA), and time difference of arrival (TDoA), can be used to estimate the distance between mobile devices. Nowadays, a lot of location systems have tried to estimate the distance between mobile devices using different models in wireless networks. For example, the Active Badge System used an infrared signal [2]. Cricket, developed at MIT, uses TDoA method [3]. Global Positioning System (GPS) uses ToA [4]. RADAR, developed at Microsoft, uses RSSI to estimate the distance [5]. SpotON is a RSSI based ad-hoc localization system [6]. In this paper, we discuss the distance estimation using Bluetooth RSSI.

The rest of the paper is organized as follows. Section II describes related work. In section III, we describe WBAN RSSI characteristic in office environment. Some concluding remarks are finally given in Section IV.

\section{RELATED WORK}

RSSI can be used to estimate the distance based on the received signal strength from another machine. The longer the distance to the receiver machine, the lesser the signal strength at received machine. Theoretically, the signal strength is inversely proportional to squared distance, and there is a known radio propagation model that is used to convert the signal strength into distance. However, in real environments, it is hard to measure distance using RSSI because of noises, obstacles, and the type of antenna. In these cases it is common to make a system calibration [7], where values of RSSI and distances are evaluated ahead of time in a controlled environment. Some experiments [8] show errors from 2 to $3 \mathrm{~m}$ in some scenarios. Distance estimation using RSSI in realworld applications is still questionable because of inaccuracy [9]. However, RSSI could become the most used technology of distance estimation from the cost/precision viewpoint because of low cost [10]. A. Awad et al. [1] presented intensively discuss and analyze approaches relying on the received signal strength indicator. It showed that even for noisy indoor environments an average positioning error of $50 \mathrm{~cm}$ on an area of $3.5 \times 4.5 \mathrm{~m}$ is possible by choosing the RF and algorithm parameters carefully based on empirical studies. S. Feldmann et al. [11] also presented an indoor positioning system based on signal strength measurements, which were approximated by the received RSSI in a mobile device.

RADAR was developed at Microsoft and used RSSI to estimate the distance [5]. It is based on an 802.11 Wireless LAN. A building-wide tracking system based on the IEEE 802.11 LAN wireless networking technology. RADAR measures the signal strength and signal-to-noise ratio at the base station, and then it computes the position within a building using these data. RADAR's scene-analysis implementation has position error within about 3 meters with 50 percent probability. 


\section{WBAN RSSI CHARACTERISTIC IN OFFICE ENVIRONMENT}

We tested the relation between the distance and Bluetooth RSSI in office environment as shown in Fig. 1. This test is performed in the direction of east $(\mathrm{E})$, west $(\mathrm{W})$, north $(\mathrm{N})$, south (S), northeast (NE), and northwest (NW).

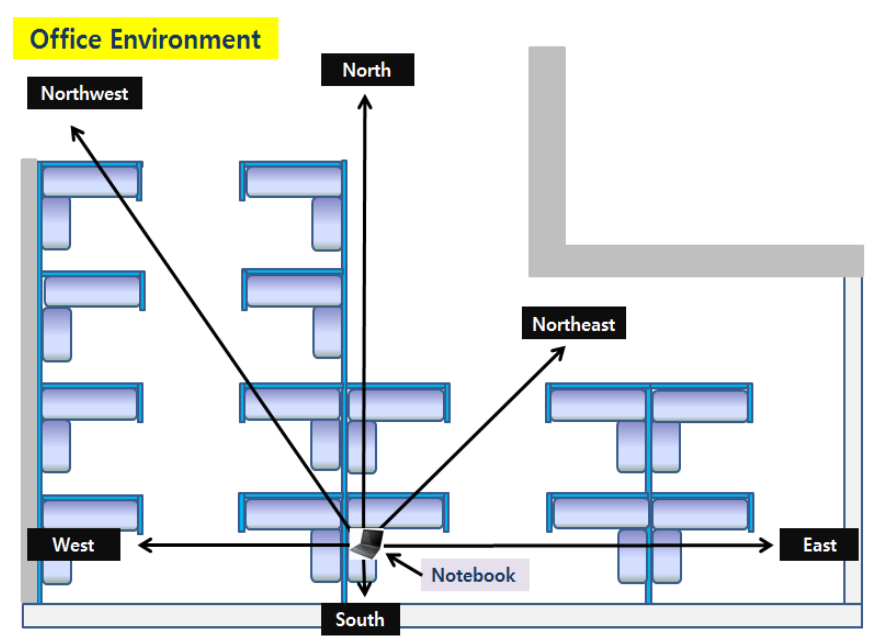

Figure 1. The office structure for testing P2P signal strength characteristic

We have measured Bluetooth RSSI with a notebook and a Nexus 7 in office environment as shown in Fig. 2. We measured 200 samples at each meter.

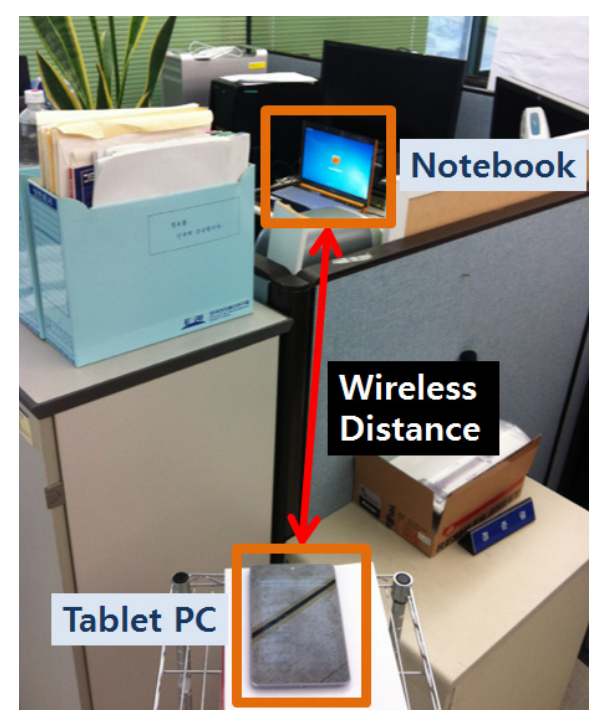

Figure 2. Bluetooth RSSI mesurement test in office environment.

The results of these experiments are shown in Fig 3. The RSSI values of east, west, south, north, northeast and northwest directions are shown in Fig. 3 (a) Fig. 3 (f), respectively. The distance estimation for recognizing WBAN area is possible with the RSSI raw data coarsely in case of north and northwest direction. However, it is hard to distinguish WBAN area in the other directions. So, we applied the low pass filter (LPF) to distinguish WBAN area more clearly. LPF equation is (1). The received signal strength $\left(T_{\mathrm{n}}\right)$ is the RSSI value receiving from the other mobile device at $n$. And the LPF value $\left(R_{\mathrm{n}}\right)$ is the RSSI value of LPF at $\mathrm{n}$. The constant $(\alpha)$ has a value that is bigger than 0 and lower than 1 .

$$
R_{n}=\alpha R_{n-1}+(1-\alpha) T_{n} .
$$

When the LPF is used, WBAN area can be distinguished more clearly as seen Fig. 3, where $(\alpha=0.9)$. There are partitions in the all directions except east direction. So, WBAN area is clearly distinguished without error in some directions. And there are a few errors in the others directions.

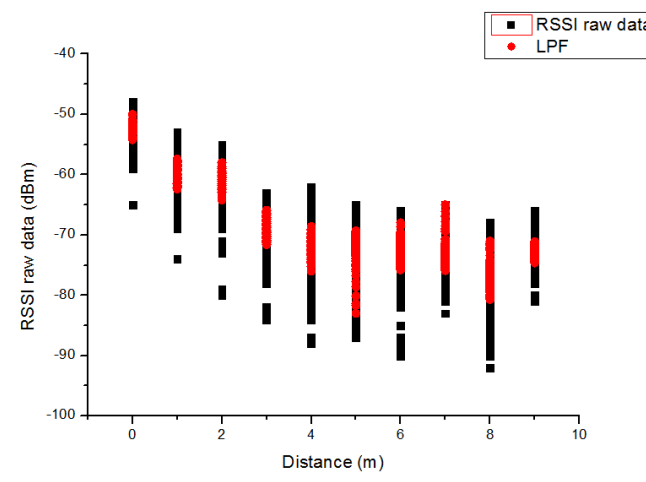

(a) RSSI value of the East direction

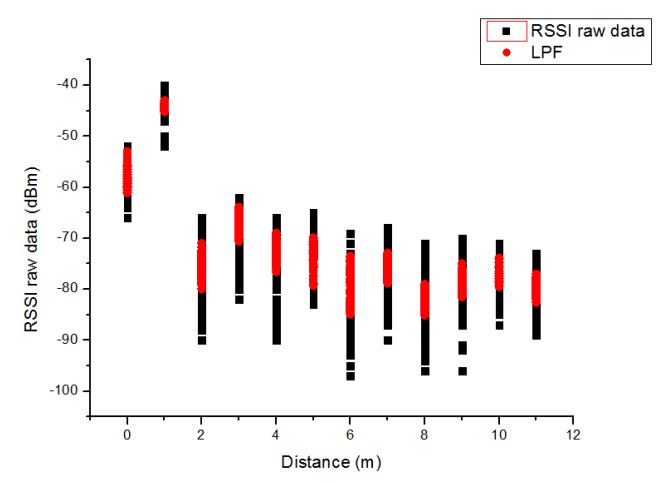

(b) RSSI value of the North direction

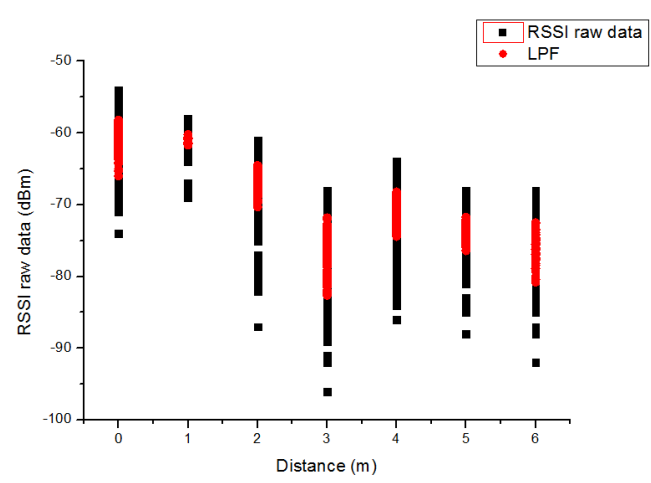

(c) RSSI value of the West direction 


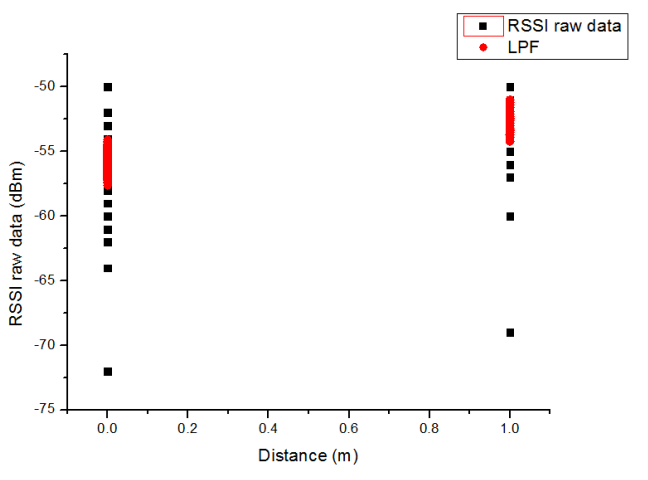

(d) RSSI value of the South direction

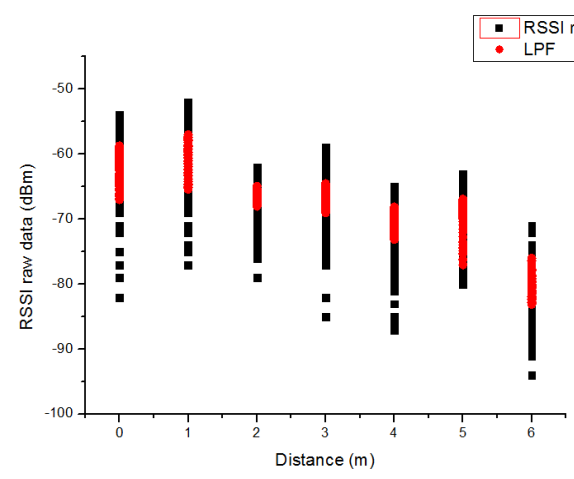

(e) RSSI value of the Northeast direction

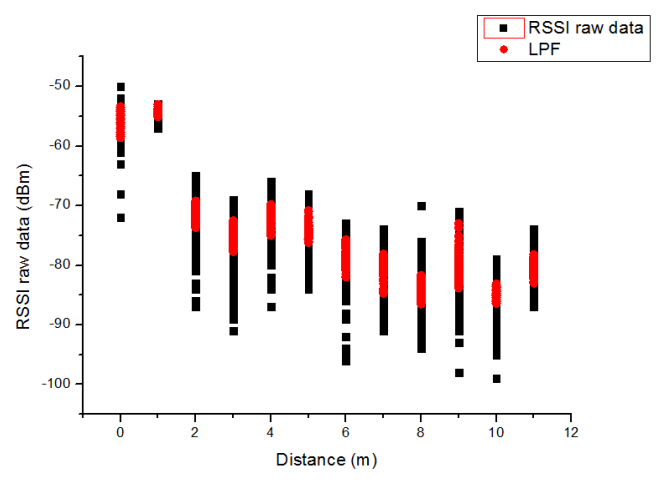

(f) RSSI value of the Northwest direction

Figure 3. The Bluetooth RSSI measurement result in office environment

We show the experimental results of northwest and east directions in Table I and Table II, respectively.

TABLE I. THE EXPERIMENTAL RESULT OF NORTHWEST DIRECTION

\begin{tabular}{|l|c|c|c|c|}
\hline \multirow{2}{*}{$\begin{array}{c}\text { Northwest } \\
\text { Direction }\end{array}$} & \multicolumn{2}{|c|}{ RSSI raw data } & \multicolumn{2}{c|}{ LPF } \\
\cline { 2 - 6 } & $\begin{array}{c}\boldsymbol{W B} \boldsymbol{A} \boldsymbol{N} \\
\text { area }\end{array}$ & $\begin{array}{c}\text { Outside of } \\
\text { WBAN } \\
\text { area }\end{array}$ & $\begin{array}{c}\text { WB } \boldsymbol{A} \boldsymbol{N} \\
\text { area }\end{array}$ & $\begin{array}{c}\text { Outside of } \\
\text { WB } \boldsymbol{A} \boldsymbol{N} \\
\text { area }\end{array}$ \\
\hline \multirow{2}{*}{ Min. value } & -72 & -99 & -58.6 & -86.5 \\
& $\mathrm{dBm}$ & $\mathrm{dBm}$ & $\mathrm{dBm}$ & $\mathrm{dBm}$ \\
\hline \multirow{2}{*}{ Max. value } & -50 & -65 & -53 & -69.2 \\
Overlap & $\mathrm{dBm}$ & $\mathrm{dBm}$ & $\mathrm{dBm}$ & $\mathrm{dBm}$ \\
\hline
\end{tabular}

TABLE II. The EXPERIMENTAL RESUlt of EAst Direction

\begin{tabular}{|l|c|c|c|c|}
\hline \multirow{2}{*}{$\begin{array}{c}\text { East } \\
\text { Direction }\end{array}$} & \multicolumn{2}{|c|}{ RSSI raw data } & \multicolumn{2}{c|}{ LPF } \\
\cline { 2 - 6 } & $\begin{array}{c}\boldsymbol{W B} \boldsymbol{A} N \\
\text { area }\end{array}$ & $\begin{array}{c}\text { Outside of } \\
\text { WBAN } \\
\text { area }\end{array}$ & $\begin{array}{c}\text { WB } \boldsymbol{A} \boldsymbol{N} \\
\text { area }\end{array}$ & $\begin{array}{c}\text { Outside of } \\
\text { WB } \boldsymbol{A} \boldsymbol{N} \\
\text { area }\end{array}$ \\
\hline \multirow{2}{*}{ Min. value } & -82 & -94 & -64.2 & -83 \\
& $\mathrm{dBm}$ & $\mathrm{dBm}$ & $\mathrm{dBm}$ & $\mathrm{dBm}$ \\
\hline \multirow{2}{*}{ Max. value } & -52 & -59 & -50 & -65.9 \\
Overlap & $\mathrm{dBm}$ & $\mathrm{dBm}$ & $\mathrm{dBm}$ & $\mathrm{dBm}$ \\
\hline
\end{tabular}

In northwest direction, when RSSI raw data is used, there is an overlap region, $7 \mathrm{dBm}$, between WBAN area and outside of WBAN area. However, when LPF data is used, there is a gap about $11.4 \mathrm{dBm}$. In east direction, when RSSI raw data is used, there is an overlap region, $23 \mathrm{dBm}$, between WBAN area and outside of WBAN area. However, when LPF data is used, there is a gap about $1.7 \mathrm{dBm}$. As the experimental results, we can distinguish about $1 \mathrm{~m}$ in case there is the partition and about $2 \mathrm{~m}$ in case there is no partition using the LPF data.

\section{IV.CONCLUSIONS}

This paper addresses the distance characteristic of Bluetooth RSSI in office environment. It is hard to classify into inside and outside of WBNA area using the RSSI raw data even if there is a partition between mobile devices. This paper shows the LPF for reducing the RSSI value deviation. The LPF data shows better result than RSSI raw data. However, LPF data need to be improved for estimating distance more exactly. So, we will design a new algorithm to estimate distance with Bluetooth RSSI.

\section{ACKNOWLEDGMENT}

This work was supported by the IT R\&D program of MSIP/KEIT, [K10041801, Zero Configuration Type Device Interaction Technology using Device Sociality between Heterogeneous Devices].

\section{REFERENCES}

[1] A. Awad, T. Frunzke, and F. Dressler, "Adaptive Distance Estimation and Localization in WSN using RSSI Measures," 10th Euromicro Conference on Digital System Design Architectures, Methods and Tools, pp. 471-478, Aug. 2007.

[2] R. Want, A. Hopper, V. Falcao, and J. Gibbons., "The Active Badge Location System," ACM Transactions on Information Systems, vol. 40, no. 1, pp. 91-102, Jan. 1992.

[3] N. B. Priyantha, A. Chakraborty, and H. Balakrishnan, "The Cricket Location-Support System,” Mobile Comp. and Networking, pp. 32-43, Aug. 2000.

[4] P. Enge and P. Misra, "Special Issue on GPS: The Global positioning System," Proc. of the IEEE, vol. 87, no. 1, pp. 3-15, Jan. 1999.

[5] P. Bahl and V. N. Padmanabhan, "RADAR: An In-Building RF-based User Location and Tracking System," Proceedings of IEEE Infocom 2000, vol. 2, pp. 75-84, Mar. 2000.

[6] J. Hightower, C. Vakili, G. Borriello, and R. Want, "Design and Calibration of the SpotON Ad-Hoc Location Sensing System," University of Washington, Seattle, Technical Report UW CSE 01-08, Aug. 2001

[7] K. Whitehouse and D. Culler, "Calibration as Parameter Estimation in Sensor Networks," Wireless Sensor Networks and Apps., pp. 59-67, 2002 . 
[8] A. Savvides, C.-C. Han, and M. B. Strivastava, "Dynamic FineGrained Localization in Ad-Hoc Networks of Sensors," 7th ACM/IEEE Int'l. Conf. Mobile Computing and Networking, Rome, Italy, pp. 166179, 2001.

[9] T. He, C. Huang, B. M. Blum, J. A. Stankovic, and T. Abdelzaher, "Range-Free Localization Schemes for Large Scale Sensor Networks," MobiCom '03, pp. 81-95, 2003.

[10] J. Bachrach and C. Taylor, "Localization in Sensor Networks," Handbook of Sensor Networks: Algorithms and Architectures, I. Stojmenovic, Ed., Wiley, Sept. 2005.

[11] S. Feldmann, K. Kyamakya, A. Zapater, and Z. Lue, "An indoor Bluetooth-based positioning system: concept, implementation and experimental evaluation," International Conference on Wireless Networks, pp. 109-113, 2003.

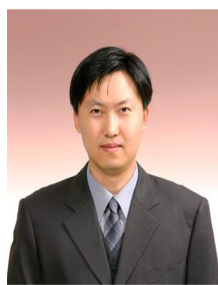

Joonyoung Jung received the B.S. and M.S. degrees in Computer Network Engineering from Soongsil University, Republic of Korea, in 1996 and 2000, respectively. Since 2000 , he has been a researcher at Electronics and Telecommunications Research Institute. He currently develops the virtualization system an cloud computing technology. His research interests in cloud computing, virtualization system, and home multimedia network service.

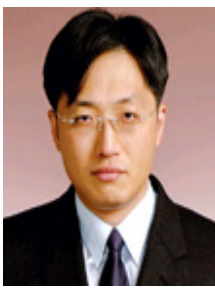

Dongoh Kang received his B.S. degree in electronic engineering from Yonsei University, Korea, in 1994. And, he received his M.S. and Ph.D. degrees in electronic engineering from Korea Advanced Institute of Science and Technology, Korea, in 1996 and 2001 respectively. Since 2001, he has been working at Electronics and Telecommunications Research Institute. His research interests include home network middleware, distributed control, and server cluster power management.

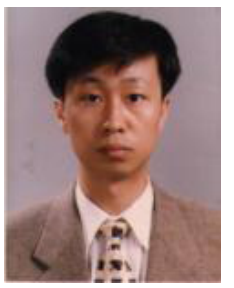

Changseok Bae received his B.S. and M.S. degrees in electronic engineering from Kyungpook National University, Korea, in 1987 and 1989 respectively. He also received his Ph.D. degree in electrical and electronic engineering from Yonsei University, Korea, in 2003. From 1989 to 1996, he was a researcher at Systems Engineering Research Institute, where he worked on image processing and pattern recognition. From 1997 to 1999, he worked with Korea Ministry of Information and Communication, where he participated in establishing national software research and development policy. Since 2000, he has been a research staff of Post-PC Platform Research Team and Personal Computing Research Team at Electronics and Telecommunications Research Institute. His research interests include image processing, multimedia codec, home server architecture, information hiding and data mining, cloud computing, social network, virtualization system. 